\title{
Days out of role and somatic, anxious-depressive, hypo-manic, and psychotic-like symptom dimensions in a community sample of young adults
}

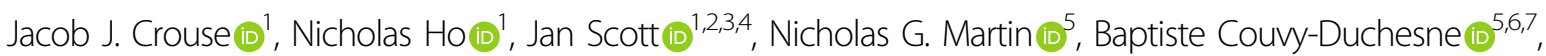

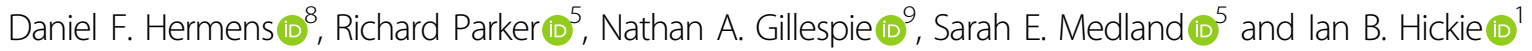

\begin{abstract}
Improving our understanding of the causes of functional impairment in young people is a major global challenge. Here, we investigated the relationships between self-reported days out of role and the total quantity and different patterns of self-reported somatic, anxious-depressive, psychotic-like, and hypomanic symptoms in a community-based cohort of young adults. We examined self-ratings of 23 symptoms ranging across the four dimensions and days out of role in >1900 young adult twins and non-twin siblings participating in the "19Up" wave of the Brisbane Longitudinal Twin Study. Adjusted prevalence ratios (APR) and 95\% confidence intervals (95\% Cl) quantified associations between impairment and different symptom patterns. Three individual symptoms showed significant associations with days out of role, with the largest association for impaired concentration. When impairment was assessed according to each symptom dimension, there was a clear stepwise relationship between the total number of somatic symptoms and the likelihood of impairment, while individuals reporting $\geq 4$ anxious-depressive symptoms or five hypomanic symptoms had greater likelihood of reporting days out of role. Furthermore, there was a stepwise relationship between the total number of undifferentiated symptoms and the likelihood of reporting days out of role. There was some suggestion of differences in the magnitude and significance of associations when the cohort was stratified according to sex, but not for age or twin status. Our findings reinforce the development of early intervention mental health frameworks and, if confirmed, support the need to consider interventions for subthreshold and/or undifferentiated syndromes for reducing disability among young people.
\end{abstract}

\section{Introduction}

The increasing number of young adults who are not in employment, education, and training is a major global challenge ${ }^{1}$. After adjusting for geopolitical variations, it is clear that depression, bipolar disorder, and schizophrenia are leading contributors to all types of functional

\footnotetext{
Correspondence: Jacob J. Crouse (jacob.crouse@sydney.edu.au)

${ }^{1}$ Youth Mental Health \& Technology Team, Brain and Mind Centre, University of Sydney, Sydney, Australia

${ }^{2}$ Academic Psychiatry, Institute of Neuroscience, Newcastle University, Newcastle, UK
}

Full list of author information is available at the end of the article impairment and social disengagement in young people ${ }^{2}$. As such, understanding the inter-relationships between clinical and functional phenomena and increasing efforts to prevent and/or modify the onset and course of mental disorders and impairment are needed ${ }^{3,4}$. Recent research demonstrates that young people who experience mental health problems and accompanying impairment often present with conditions that do not meet diagnostic criteria for a full-threshold episode of a mental disorder, but with so-called "attenuated" or "subthreshold" syndromes or with undifferentiated symptoms that impair day to day functioning ${ }^{5-7}$. Despite this, there are few studies that

\section{(c) The Author(s) 2021}

(c) (i) Open Access This article is licensed under a Creative Commons Attribution 4.0 International License, which permits use, sharing, adaptation, distribution and reproduction cc) in any medium or format, as long as you give appropriate credit to the original author(s) and the source, provide a link to the Creative Commons license, and indicate if changes were made. The images or other third party material in this article are included in the article's Creative Commons license, unless indicated otherwise in a credit line to the material. If material is not included in the article's Creative Commons license and your intended use is not permitted by statutory regulation or exceeds the permitted use, you will need to obtain permission directly from the copyright holder. To view a copy of this license, visit http://creativecommons.org/licenses/by/4.0/. 
examine the relationships between impairment and these broader dimensions of psychopathology. Given the emphasis on public health programs and early intervention services that target young people in the early stages of mental illness, it is important to clarify whether and how individual symptoms, different symptom patterns or dimensions, and/or total symptom load are linked with functioning.

Clinical and community studies over the last 30 years have provided support for dimensional models of psychopathology, and the notion that the magnitude of functional impairment is often associated with incremental increases in symptom load or burden. Most studies to date have focused on psychological symptoms (such as anxious-depressive, hypomanic, or psychotic-like experiences) or on somatic (physical) symptoms. For example, a study of adults in the Epidemiological Catchment Area study showed that people experiencing minor depression with mood disturbance (based on the Diagnostic Interview Schedule) had a 1.6-fold greater risk of self-reported disability days compared to asymptomatic individuals, while those with major depression had a 4.8-fold greater risk of disability days ${ }^{8}$. Further, given its higher relative prevalence, minor depression was associated with $51 \%$ more days out of role than major depression $^{8}$. Likewise, self-reported somatic symptoms such as sleep-wake disturbances are common in general population samples and are associated with high rates of self-reported disability ${ }^{9,10}$. For example, a primary carebased study of 15 common physical symptoms in adults (e.g., fatigue, joint or limb pain, and headache) reported that all self-reported symptoms were significantly associated with self-reported impairment, and that symptom load (the total number of symptoms) was strongly associated with functional status ${ }^{11}$. Psychotic experiences and attenuated psychotic syndromes have received increased attention in recent years following recognition of their higher-than-expected prevalence in the community and relationships with impairment. For example, two partially overlapping studies of high school and university students demonstrated that several subtypes of self-reported psychotic-like experiences (bizarre experiences, perceptual abnormalities, persecutory ideas, and grandiosity) were each associated with impaired self-reported functioning $^{12,13}$, and clinical studies of non-psychotic help-seeking young people accessing youth mental health services have reported similar relationships between self- and clinician-rated impairment and self-reported bizarre experiences and persecutory ideas ${ }^{14}$. Only a handful of studies have examined relationships between impairment and hypo-manic symptoms and syndromes. A re-analysis of five sites of the Epidemiological Catchment Area study showed that Diagnostic Interview Schedule ascertained "subthreshold manic/hypomanic symptoms" (which together represent a similar condition to brief hypomania meeting the Zurich criteria) were associated with increased need for welfare/disability benefits ${ }^{15}$, and two studies of community-based cohorts of young people showed that individuals with clinically-determined subthreshold bipolar disorder in adolescence had greater selfrated and clinician-rated functional impairment in early adulthood compared to asymptomatic individuals ${ }^{16,17}$. Altogether, these and other studies demonstrate incremental relationships between a continuum of psychopathology and the level of impairment ${ }^{18-23}$.

An important limitation of previous studies has been their focus on single symptom dimensions or syndromes (i.e., they do not explore a broad range of somatic and psychological symptoms in conjunction). Recently, we showed that subthreshold presentations of depression-like, hypomanic-like, and psychotic-like syndromes were each associated with greater level of perceived impairment in young adults compared to those with no subthreshold syndrome and that experiencing $>1$ subthreshold syndrome was associated with even greater impairment ${ }^{24}$. As many young people experience non-specific admixtures of symptoms in the early stages of mental illness ${ }^{25}$, and comorbidity is the rule rather than the exception in young people $^{26}$, there is a need to examine broader relationships of impairment and patterns of symptoms occurring both within specific dimensions and across multiple dimensions ${ }^{27,28}$.

Accordingly, the goal of this study is to model the associations between impairment and levels of symptoms within and across somatic, anxious-depressive, hypomanic, and psychotic-like dimensions in a large community-based cohort of twins and non-twin siblings (Brisbane Longitudinal Twin Study). Here, we selected "days out of role" as a measure of impairment because of its wide use (e.g., WHO World Mental Health surveys), convenience, objectivity, and established links with mental health across a range of cultures, countries, and clinical and non-clinical settings ${ }^{6,8,29-33}$. Specifically, the current study examines the relationships between self-rated days out of role and:

1. Individual self-reported symptoms (i.e., somatic, anxious-depressive, hypomanic, and/or psychoticlike experiences, irrespective of symptom dimension).

2. Total number of self-reported symptoms within each dimension.

3. Total number of undifferentiated symptoms (i.e., across the four dimensions).

\section{Subjects and methods}

\section{Ethical approval and observational study reporting}

Ethical approval was obtained from the Human

Research Ethics Committee at the Queensland Institute of Medical Research for all Brisbane Longitudinal Twin 
Study research projects (reference numbers: EC00278, P1212). This study follows the Strengthening the Reporting of Observational Studies in Epidemiology (STROBE) guidelines ${ }^{34}$ (see STROBE checklist in Supplementary Materials). Written, informed consent was obtained from participants, and their parents if applicable (i.e., participants aged under 18).

\section{Study participants}

The Brisbane Longitudinal Twin Study (BLTS) is an ongoing prospective cohort study of twins and their siblings in Queensland, Australia, conducted at the Queensland Institute of Medical Research (QIMR). The BLTS began in 1992, recruiting twins around age 12 from primary and secondary schools in the greater Brisbane area in Queensland via media appeals, word of mouth, or from the Australian Twin Registry ${ }^{35,36}$. The BLTS was originally conceived to study the development of melanocytic naevi (moles) and cognition during adolescence, but additionally includes a rich variety of biological, psychological, and behavioral assessments of personality, substance use, neurobiology, acne, and taste and olfaction, among others ${ }^{35,37-39}$. As described elsewhere ${ }^{35,38}$, the BLTS cohort appears representative regarding psychometric IQ, the proportions of twins by sex and zygosity are in keeping with population expectations, and the ethnic distribution reflects the population structure of Queensland at the time of recruitment (mostly European ancestry and minorities of Asian ancestry). All BLTS cohort members have been invited to participate in reassessments around age 14, 16, 19, and most recently, $25^{40}$. The current study focuses on the "Nineteen and Up" (19Up) study, for which data were collected between $2009-2016^{37}$ (a flow diagram is available in Supplementary Fig 1).

\section{Eligibility criteria}

Prospective recruits were ineligible for initial entry into the BLTS (i.e., around age 12) if they had: (1) vision/hearing impairments; (2) history of closed head injury; (3) or significant mental or neurological disorders. For this study, participants were eligible if they responded to self-report questionnaires of mental health symptoms and functioning in the "19Up" wave. For participants who completed questionnaires on multiple occasions in 19Up, we selected their most recent responses. There were no age restrictions set for inclusion in the current study.

\section{Assessments \\ Sociodemographic characteristics}

Data regarding age, sex, zygosity, marital status, occupation, and highest level of education were collected using self-report questionnaires.

\section{Self-rated symptoms}

a. Somatic and anxious-depressive symptoms: Measured using the 6-item SOMA and 6-item PSYCH subscales of the 12-item Somatic and Psychological Health Report (SPHERE-12) ${ }^{32}$. The SOMA-6 subscale includes a subset of six items from the Schedule of Fatigue and Anergia (SOFA) ${ }^{41}$, which were initially included based on their ability to predict a SOFA case $^{32}$. The PSYCH-6 subscale includes a subset of six items from the General Health Questionnaire $(\mathrm{GHQ})^{42}$, initially included based on their being from the depression/anxiety domain and their ability to predict a mental disorder on the GHQ ${ }^{32}$. The SPHERE-12 subscales have high internal consistency (Cronbach's $\alpha$ : PSYCH- $6=$ $0.90 ; \quad$ SOMA- $6=0.80$ ) and high test-retest reliability over two occasions 3-6 months apart in a general practice sample (intraclass correlation: PSYCH $-6=0.81 ; \quad$ SOMA- $6=0.80)^{32}$. For each symptom item, participants were required to answer the question "Over the past few weeks have you been troubled by..." with one of three responses: "never or some of the time", "a good part of the time", or "most of the time". We coded these symptoms as present (1) ("a good part of the time" or "most of the time") or absent (0) ("never or some of the time"). The items are shown in Fig. 1 and a full list of questions is in Supplementary Table 1.

b. Hypomanic symptoms: Measured by five items partially derived from symptoms included in the DSM-IV, bipolar at-risk criteria ${ }^{43,44}$, and Altman SelfRating Mania Scale ${ }^{45}$. For each item, participants were required to answer yes/no to the question "Have you ever experienced a definite period where for more than 2 or 3 days you...". These items are useful for assessing hypomanic symptoms/syndromes or bipolar spectrum conditions in young people ${ }^{46}$, which are more prevalent in the general population than previously thought ${ }^{47,48}$. These items are shown in Fig. 1 and a full list of questions is in Supplementary Table 1.

c. Psychotic-like symptoms: Measured by six items of positive psychotic experiences that are prevalent in clinical and community-based samples of young people and related to impairment ${ }^{12-14}$. For each item, participants were required to answer yes/no to the question: "Have you ever...". The six items are shown in Fig. 1 and a full list of item questions is in Supplementary Table 1.

\section{Self-rated days out of role}

Days out of role was measured using a modified version of the World Health Organization's Disability Assessment 


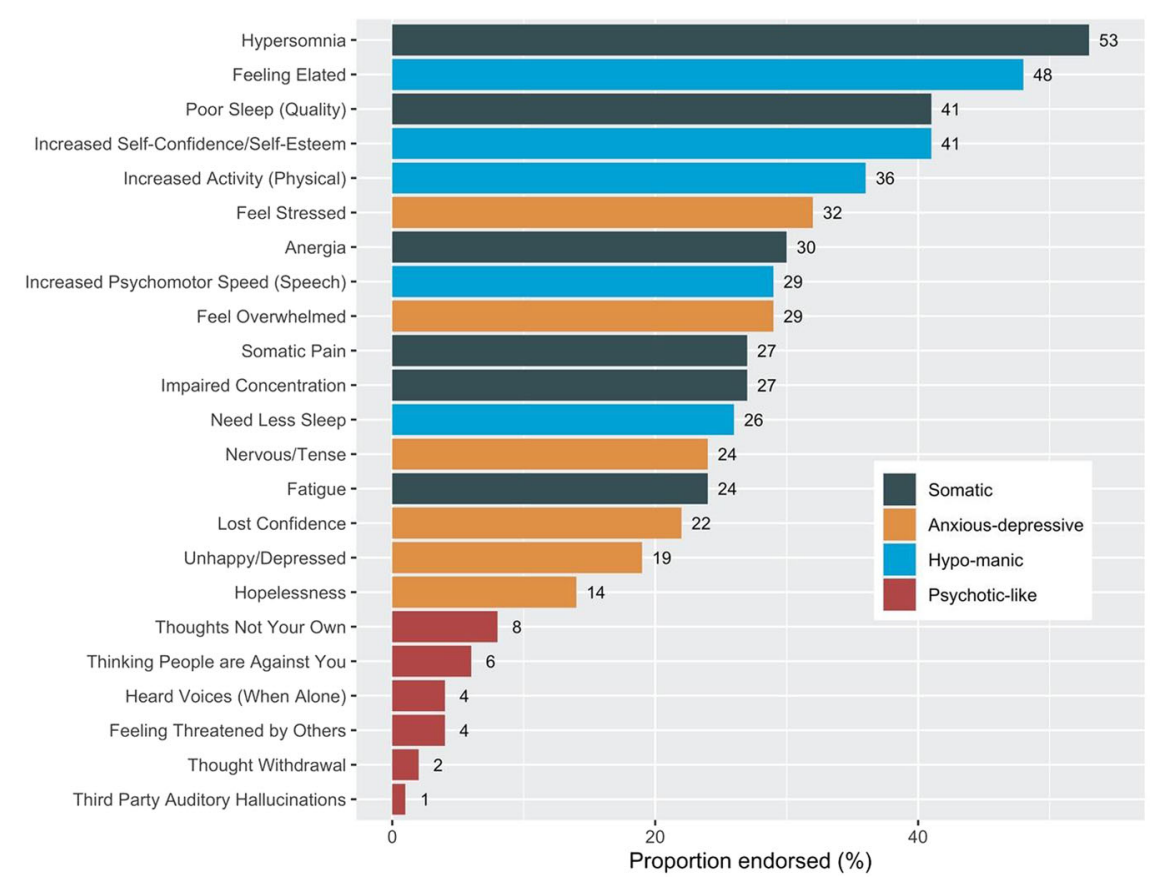

Fig. 1 Prevalence of self-rated somatic, anxious-depressive, hypomanic, and psychotic-like symptoms in a community-based cohort of young adults. Percentages are reported to the nearest whole number.

Schedule (WHO-DAS) ${ }^{49}$. Participants were asked: "During the last few weeks how many days in total were you unable to carry out your usual daily activities fully?" Previous work has reported good concordance with a similarly worded question and payroll records of employed people ${ }^{50}$. Here, participants were classified as having any days out of role if they responded with $\geq 1$.

\section{Statistical analyses}

Analyses were conducted using R (version 4.02) with the RStudio $\mathrm{IDE}^{51}$ and run on macOS Catalina (version 10.15.7). Continuous measures are reported as means and standard deviations and binary measures as numbers and percentages. Complete case analysis was used. Our main analyses included three models: (1) absence/presence of 23 individual symptoms and age, sex, and twin status; (2) total number of symptoms within four domains (anxiousdepressive, somatic, hypomanic, and psychotic-like) and age, sex, and twin status; (3) total number of "undifferentiated" symptoms and age, sex, and twin status. In exploratory analyses, we re-ran each model separately in males and females. We calculated adjusted prevalence ratios (APRs) as a measure of association between the predictor and outcome variables. Adjusted prevalence ratios have been reported to be preferred over odds ratios for clustered, cross-sectional epidemiological (e.g., twins and siblings) and samples with common outcomes (e.g., $>10 \%)^{52}$. Using the "prLogistic" package (version 1.2), we estimated APRs using random effects logistic regression models and estimated 95\% confidence intervals (95\% CI) using the "delta" method, which produces an approximate standard error for the APR to estimate the $95 \% \mathrm{CI}^{52,53}$. To control for multiple comparisons in our main analyses, we report false discovery rate (FDR) $P$-values alongside raw $P$-values, calculated using the Benjamini-Hochberg procedure ${ }^{54}$. We reserve use of "significant" for coefficients with an FDR-adjusted $P$-value below $<0.05$. Analyses were conducted by J.J.C. and N.H. between May and September 2020.

\section{Results}

\section{Sample characteristics}

Of 2773 individuals who participated in the "19Up" wave of the BLTS ${ }^{37}$, a total of 1904 individuals met eligibility criteria for this study, and sociodemographic characteristics are in Table 1. Of these 1904 participants, 791 were male and 1113 were female. The median age was $26(\mathrm{IQR}=$ 23-29). There were 605 monozygotic twin individuals, 753 dizygotic twin individuals, and 545 non-twin siblings (zygosity was undetermined for one individual).

The sample self-reported a cumulative total of 2310 days out of role in the few weeks prior to assessment. Over onequarter reported at least one day out of role $(28 \%, 540 /$ 1904). Females were more likely to report one or more days out of role than males $(32.2 \%$ vs. $23.0 \%)\left(\chi^{2}=18.63, \mathrm{df}=1\right.$, $P<0.001)$. The prevalence of self-rated somatic, anxiousdepressive, psychotic-like, and hypomanic symptoms 
Table 1 Sociodemographic characteristics of sample $(N=$ 1904).

\begin{tabular}{lc}
\hline & $\boldsymbol{N}$ (\%) or $\boldsymbol{M}$ (SD) \\
\hline Age, years & $26.3(3.9)$ \\
Sex (female) & $1113(58.5 \%)$ \\
Marital status ${ }^{\mathrm{a}}$ & \\
Married & $466(24.5)$ \\
Separated, divorced, and widowed & $41(2.2)$ \\
Never married & $1395(73.3)$ \\
Primary occupation & \\
Full-time & $1184(62.3)$ \\
Part-time & $238(12.5)$ \\
Studying & $252(13.2)$ \\
Home duties & $106(5.6)$ \\
Employed, not working (e.g., illness) & $28(1.5)$ \\
Receiving sickness/disability benefits & $16(0.8)$ \\
Volunteer & $10(0.5)$ \\
Unemployed & $62(3.3)$ \\
Prefer not to answer/don't know & $6(0.3)$ \\
Education (highest level) & \\
Postgraduate degree & \\
Undergraduate degree & $275(14.5)$ \\
Certificate/diploma & $484(25.4)$ \\
Junior/senior high school & $299(15.7)$ \\
No formal education & $1(0.1)$ \\
\hline & \\
\hline
\end{tabular}

${ }^{\mathrm{a}}$ Missing for $N=2$.

ranged from 1 to $53 \%$ (Fig. 1). The five most prevalent symptoms were "hypersomnia" (53\%), "feeling elated" (48\%), "increased self-confidence/self-esteem" (41\%), "poor sleep (quality)" (41\%), and "increased activity (physical)" (36\%). The five least prevalent were all psychotic-like symptoms. Participants endorsed between 0-22 symptoms, with a median of five symptoms (see Supplementary Table 2 for the proportions of participants endorsing 0-22 symptoms).

\section{Days out of role and individual symptoms}

Our first set of models examined the relationships between days out of role and the 23 individual symptom items (see Supplementary Table 3). In the total sample, participants had a significantly increased likelihood of having days out of role if they endorsed impaired concentration $(\mathrm{APR}=1.59)$, increased psychomotor speed (speech) (APR $=1.40)$, and fatigue $(\mathrm{APR}=1.34)$. Hypersomnia $(\mathrm{APR}=$
1.27) and hopelessness (APR $=1.36)$ were no longer significantly associated with having days out of role after adjusting for multiple comparisons.

\section{Days out of role and total number of somatic, anxious-} depressive, hypomanic, and psychotic-like symptoms

Our second set of models examined the relationships between days out of role and the number of symptoms in each dimension (Note: the psychotic-like symptom dimension was truncated at $\geq 2$ symptoms due to the distribution of self-ratings; see Supplementary Table 4). In the total sample (Fig. 2A and Table 2), there were significant stepwise relationships between likelihood of having days out of role and all levels of the somatic dimension: one somatic symptom $(\mathrm{APR}=1.90)$, two somatic symptoms $(\mathrm{APR}=2.07)$, three somatic symptoms $(\mathrm{APR}=2.34)$, four somatic symptoms $(\mathrm{APR}=2.72)$, five somatic symptoms $(\mathrm{APR}=3.18)$, and six somatic symptoms $(\mathrm{APR}=3.67)$. The patterns of association for days out of role and anxious-depressive symptoms was somewhat curvilinear: two anxious-depressive symptoms $(\mathrm{APR}=1.39)$ (however this was no longer significant when adjusting for multiple comparisons), four anxious-depressive symptoms (APR $=$ $1.89)$, five anxious-depressive symptoms ( $\mathrm{APR}=2.60)$, and six anxious-depressive symptoms (APR $=2.83$ ). Finally, endorsing $\geq 2$ psychotic-like symptoms was not significantly associated with having days out of role after adjusting for multiple comparisons ( $\mathrm{APR}=1.54$ ), while endorsing five hypomanic symptoms $(\mathrm{APR}=1.71)$ was associated with having days out of role. As with the individual symptoms model, there was some suggestion of sex differences (Fig. 2B).

\section{Days out of role and total number of undifferentiated symptoms}

Our third and final set of models examined the relationships between days out of role and the total number of undifferentiated symptoms across the four dimensions (Note: the total number of symptoms was truncated at $\geq 15$ symptoms due to the distribution of symptom ratings; see Supplementary Table 2). About $85 \%$ of the sample endorsed at least one symptom. In the total sample (Fig. 3A and Table 3), there was a linear relationship between having any days out of role and endorsing $\geq 2$ symptoms, for example: two symptoms $(\mathrm{APR}=1.94)$, 6 symptoms $(\mathrm{APR}=2.98), 10$ symptoms $(\mathrm{APR}=4.34)$, 14 symptoms $(\mathrm{APR}=6.18)$, and $15+$ symptoms $(\mathrm{APR}=$ 7.41). Again, there was some suggestion of sex differences (Fig. 3B).

\section{Discussion}

In this community-based cohort of young adults, we identified both general and specific relationships between the likelihood of having $\geq 1$ day out of role and 

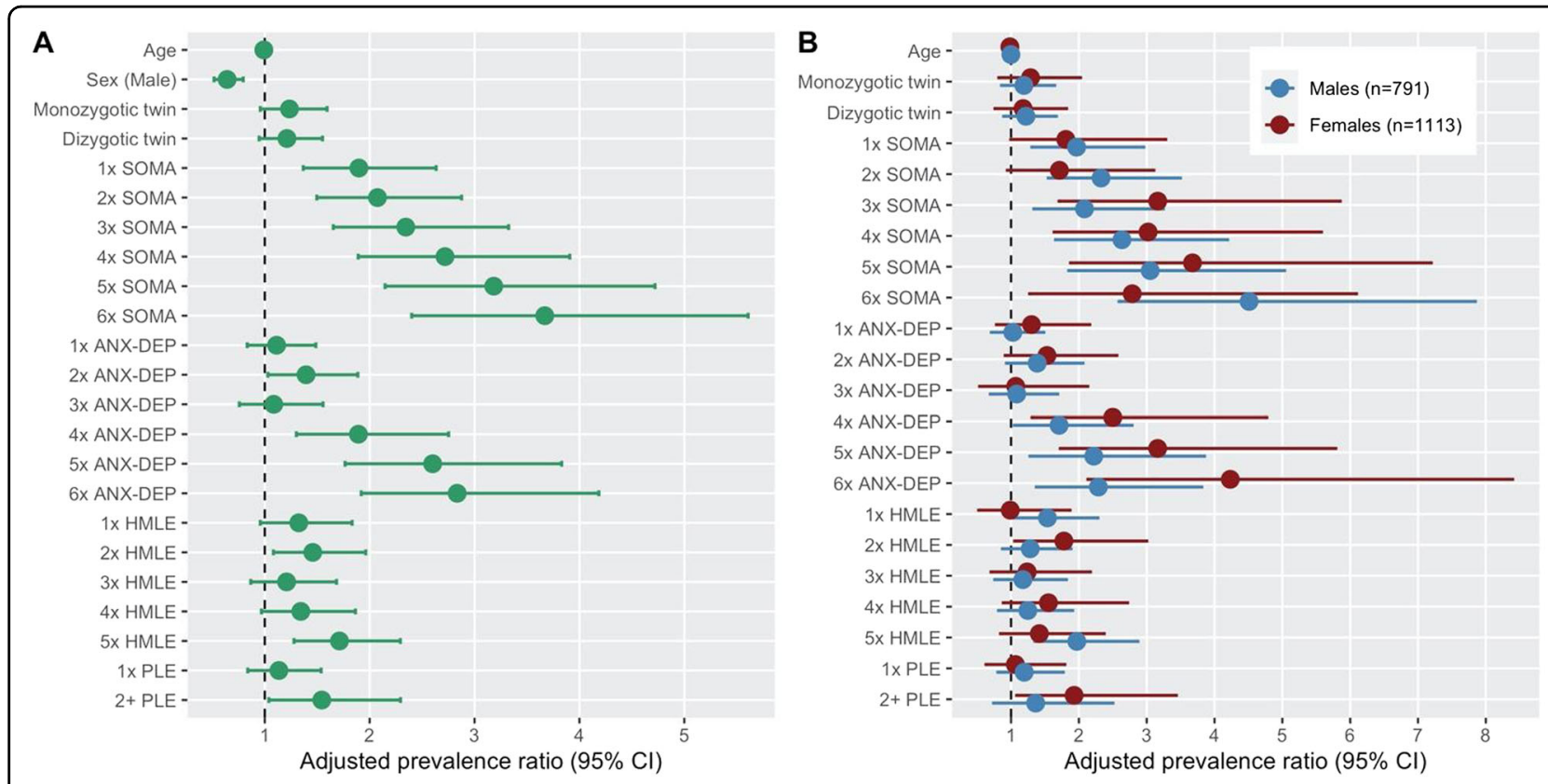

Fig. 2 Relationships between days out of role and the number of somatic, anxious-depressive, hypo-manic, and psychotic-like symptoms in a community-based cohort of young adults. A Model for total sample; $\mathbf{B}$ separate models stratified by sex. SOMA somatic symptoms, ANX-DEP = anxious-depressive symptoms, HMLE hypomanic, PLE psychotic-like symptoms.

experiencing symptoms associated with mental ill-health. Before interpreting our findings, it is worth qualifying first that symptoms and impairment were self-rated in this study, and these relationships may not be equivalent to those that would be observed if symptoms and impairment were rated by trained clinicians or other observers; however, many previous studies in this area relied similarly on self-ratings ${ }^{9-13}$. Nonetheless, we focus this discussion on three key observations. First, specific symptoms including hypersomnia, impaired concentration, fatigue, impaired sleep quality, and increased psychomotor speed (speech) were each associated with having days out of role, with more consistent associations noted for hypersomnia and impaired concentration (i.e., significant in the total sample and one of the sex-stratified models). Second, somatic symptoms were a strong predictor of having days out of role. Specifically, endorsing at least one somatic symptom was associated with elevated likelihood of having days out of role, and there was a stepwise relationship between an increasing number of somatic symptoms and the likelihood of having days out of role. Finally, there was a clear stepwise relationship between total undifferentiated symptom burden (including somatic, anxious-depressive, hypomanic, and psychotic-like experiences, irrespective of symptom dimension) and the likelihood of having any days out of role.

To begin with, our finding that specific somatic symptoms (e.g., hypersomnia) and the total number of somatic symptoms were predictors of impairment has high face validity, and adds to the growing body evidence from clinical and community-based studies demonstrating a strong and robust link between impairment and somatic symptoms and syndromes across the lifespan ${ }^{11,32,55-58}$. The likelihood of having days out of role increased linearly with endorsement of one to six somatic symptoms (relative to none) and the APRs between impairment, and the total number of somatic symptoms was consistently higher compared to an equivalent number of symptoms from the other dimensions. For example, while there was a significant relationship between impairment and reporting $\geq 4$ anxious-depressive symptoms, the likelihood of impairment was about the same for 4 anxiousdepressive symptoms $(\mathrm{APR}=1.89)$ as a single somatic symptom (APR $=1.90)$. Similarly, we found that participants had to endorse all five hypo-manic symptoms before likelihood of impairment was statistically significant. While some studies have reported associations between hypomanic symptoms and impairment ${ }^{15}$, other studies of bipolar disorders or transdiagnostic samples tend to show stronger relative associations with impairment for depressive compared to small numbers of hypomanic symptoms ${ }^{59-61}$. Finally, consistent with clinical and community-based studies, participants endorsing psychotic-like symptoms had elevated likelihood of impairment ${ }^{62,63}$, however, this finding was not statistically significant when adjusting for multiple comparisons. These associations regarding the number of hypomanic and psychotic-like symptoms mirror the findings of our 
Table 2 Relationships between somatic, anxiousdepressive hypo-manic, and psychotic-like, symptoms and "days out of role" in a community sample of young adults.

\begin{tabular}{|c|c|c|c|}
\hline $\begin{array}{l}\text { Symptom } \\
\text { dimension }\end{array}$ & $\begin{array}{l}\text { APR Whole } \\
\text { sample }\end{array}$ & $\begin{array}{l}\text { Raw } p \text { - } \\
\text { value }\end{array}$ & $\begin{array}{l}\text { FDR-adjusted } p \text { - } \\
\text { value }\end{array}$ \\
\hline \multicolumn{4}{|l|}{ Somatic } \\
\hline 0 & 1.00 & & \\
\hline 1 & $1.90(1.37-2.63)$ & $* * *$ & $* * *$ \\
\hline 2 & $2.07(1.50-2.88)$ & $* * *$ & $* * *$ \\
\hline 3 & $2.34(1.65-3.32)$ & $* * *$ & $* * *$ \\
\hline 4 & $2.72(1.89-3.91)$ & $* * *$ & $* * *$ \\
\hline 5 & $3.18(2.15-4.72)$ & $* * *$ & $* * *$ \\
\hline 6 & $3.67(2.40-5.61)$ & $* * *$ & $* * *$ \\
\hline \multicolumn{4}{|c|}{ Anxious-depressive } \\
\hline 0 & 1.00 & & \\
\hline 1 & $1.11(0.83-1.49)$ & NS & NS \\
\hline 2 & $1.39(1.03-1.89)$ & $*$ & NS \\
\hline 3 & $1.09(0.76-1.56)$ & NS & NS \\
\hline 4 & $1.89(1.30-2.75)$ & $* * *$ & $* *$ \\
\hline 5 & $2.60(1.77-3.83)$ & $* * *$ & $* * *$ \\
\hline 6 & $2.83(1.92-4.19)$ & $* * *$ & $* * *$ \\
\hline \multicolumn{4}{|l|}{ Hypomanic } \\
\hline 0 & 1.00 & & \\
\hline 1 & $1.32(0.96-1.83)$ & NS & NS \\
\hline 2 & $1.46(1.08-1.96)$ & * & * \\
\hline 3 & $1.21(0.87-1.68)$ & NS & NS \\
\hline 4 & $1.34(0.97-1.86)$ & NS & NS \\
\hline 5 & $1.71(1.28-2.29)$ & $* * *$ & $* * *$ \\
\hline \multicolumn{4}{|l|}{ Psychotic-like } \\
\hline 0 & 1.00 & & \\
\hline 1 & $1.13(0.84-1.54)$ & NS & NS \\
\hline $2+$ & $1.54(1.04-2.29)$ & * & NS \\
\hline Age & $0.99(0.97-1.02)$ & NS & NS \\
\hline \multicolumn{4}{|l|}{ Sex } \\
\hline Female & 1.00 & & \\
\hline Male & $0.64(0.52-0.79)$ & $* * *$ & $* * *$ \\
\hline \multicolumn{4}{|l|}{ Twin status } \\
\hline Not a twin & 1.00 & & \\
\hline Monozygotic & $1.24(0.96-1.59)$ & NS & NS \\
\hline Dizygotic & $1.21(0.94-1.55)$ & NS & NS \\
\hline
\end{tabular}

APR adjusted prevalence ratio, NS non-significant $(p>0.05)$. ${ }^{*} p<0.05$.

${ }^{* *} p<0.01$.

${ }_{* * *}^{*} p<0.001$.

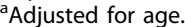

${ }^{\mathrm{b}}$ Adjusted for zygosity.

${ }^{\mathrm{C}}$ Adjusted for sex.

previous study in this sample, in which we reported significant associations between the perceived level of functional impairment associated with self-reported mental health problems and the presence of a hypomanic or psychotic-like subthreshold syndrome (operationalised as $\geq 5$ hypomanic or $\geq 2$ psychotic-like symptoms, respectively) ${ }^{24}$. Altogether, we interpret these findings to indicate that a lower threshold of somatic symptoms is sufficient to precipitate impaired role functioning (if we assume a causal relationship), while crossing a higher threshold for anxious-depressive, hypomanic, and psychotic-like symptoms is necessary to produce impairment.

\section{Limitations}

Our findings should be interpreted in the context of several limitations. First, participants self-reported their symptoms and impairment, and we did not model the likely impacts of severity and persistence of symptoms on impairment ${ }^{58}$. Second, somatic and anxious-depressive symptoms were measured "over the past few weeks", whereas hypo-manic and psychotic-like symptoms were lifetime questions ("Have you ever..."); these differences may partly account for the lower relative associations between hypomanic and psychotic-like symptoms. Third, we cannot conclude whether somatic symptoms were medically explained or not, which has implications for their management ${ }^{64,65}$. Fourth, while we adjusted our models for twin status and calculated APRs as a recommended measure of association for cross-sectional, clustered data ${ }^{52}$, we treated our study participants as singletons and interpret the findings at the individual level rather than the family or twin-pair level. Fifth, this study was cross-sectional, and we cannot truly assume the direction of causality of any association.

\section{Potential implications}

There are several potential implications of this study. First, somatic symptoms were common (endorsed by 24-53\% of the sample) and the stepwise relationship between the total number of somatic symptoms, and the likelihood of impairment suggests that reducing specific symptoms or the total symptom load could help alleviate impairment. Like their older adult counterparts, young adults with impairing somatic symptoms may benefit from psychological therapies such as cognitive behavior therapy $^{66}$. Speculatively, lifestyle modifications with potentially "broad spectrum" benefits, such as increasing exercise or improving sleep-wake cycles, might improve specific (e.g., hypersomnia, impaired concentration) and/or overall somatic symptoms ${ }^{67-69}$. Second, our finding that the total number of undifferentiated symptoms (irrespective of dimension) showed a strong linear association with impairment is a novel finding in this type of sample. As above, it is possible that encouraging lifestyle modifications that are have been shown to improve a range of mental health symptoms, such as improving sleep disturbance and/or sleep-wake cycles ${ }^{70-72}$, could reduce the overall burden of symptoms and partially alleviate associated impairment. Importantly, these types of lifestyle modifications and related interventions are scalable, cost-effective, and engagement can be encouraged via public health programs or increasingly popular digital technologies (e.g., wearable activity/sleep monitors) ${ }^{73,74}$. 

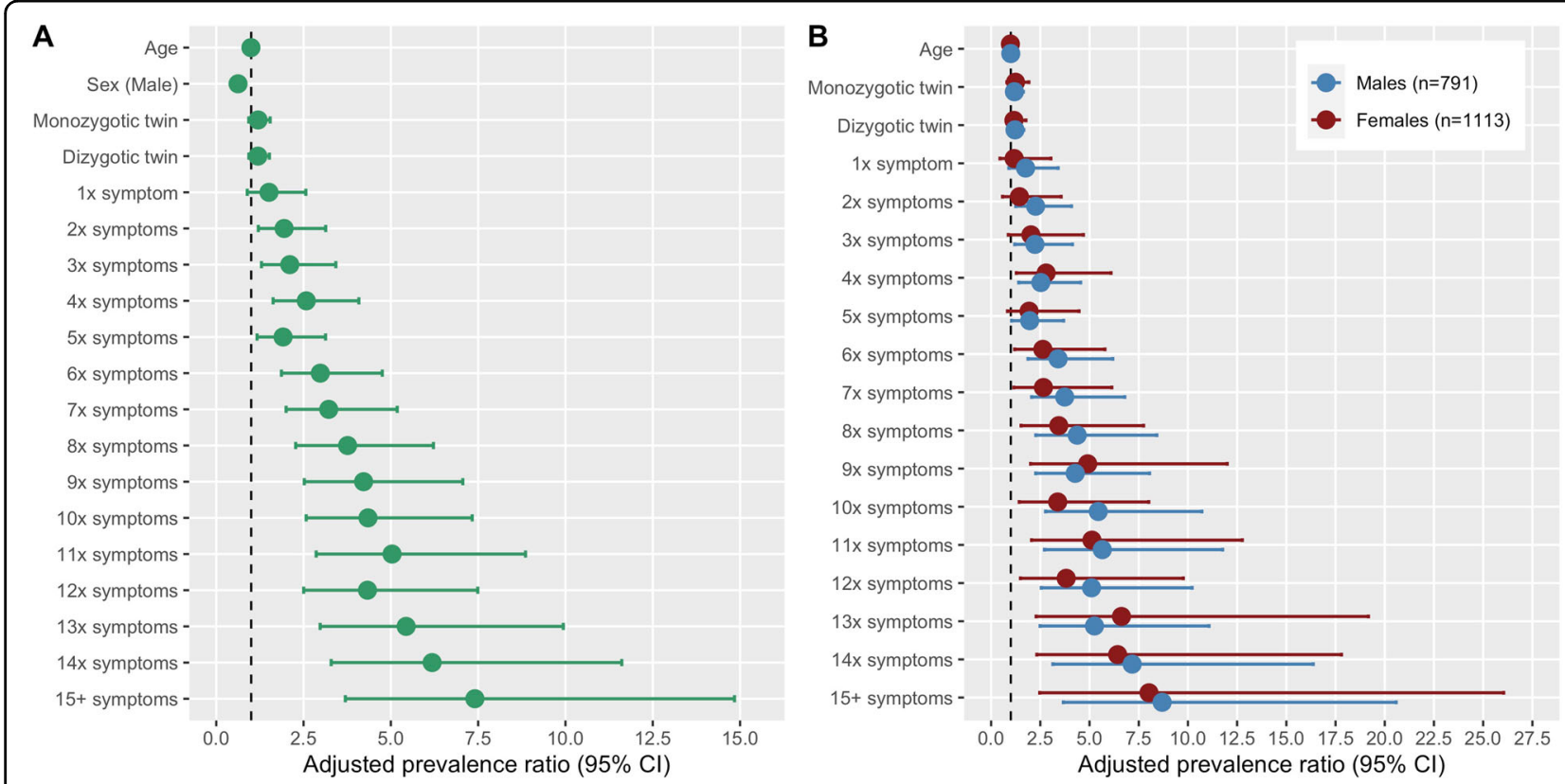

Fig. 3 Relationships between days out of role and the total number of undifferentiated symptoms in a community-based cohort of young adults. A Model for total sample. B Separate models stratified by sex.

Table 3 Relationships between number of "undifferentiated" symptoms and "days out of role" in a community sample of young adults.

\begin{tabular}{|c|c|c|c|}
\hline & APR whole sample ${ }^{a, b, c}$ & Raw $p$-value & FDR-adjusted $p$-value \\
\hline \multicolumn{4}{|c|}{ Number of symptoms } \\
\hline 0 & 1.00 & & \\
\hline 1 & $1.51(0.89-2.57)$ & NS & NS \\
\hline 2 & $1.94(1.20-3.13)$ & $* *$ & $*$ \\
\hline 3 & $2.11(1.30-3.42)$ & $* *$ & $* *$ \\
\hline 4 & $2.58(1.63-4.08)$ & $* * *$ & $* * *$ \\
\hline 5 & $1.91(1.17-3.13)$ & $* *$ & * \\
\hline 6 & $2.98(1.87-4.75)$ & $* * *$ & $* * *$ \\
\hline 7 & $3.22(2.00-5.18)$ & $* * *$ & $* * *$ \\
\hline 8 & $3.76(2.27-6.22)$ & $* * *$ & $* * *$ \\
\hline 9 & $4.22(2.52-7.06)$ & $* * *$ & $* * *$ \\
\hline 10 & $4.34(2.58-7.33)$ & $* * *$ & $* * *$ \\
\hline 11 & $5.03(2.86-8.86)$ & $* * *$ & $* * *$ \\
\hline 12 & $4.33(2.50-7.49)$ & $* * *$ & $* * *$ \\
\hline 13 & $5.44(2.98-9.94)$ & $* * *$ & $* * *$ \\
\hline 14 & $6.18(3.29-11.61)$ & $* * *$ & $* * *$ \\
\hline $15+$ & $7.41(3.70-14.84)$ & $* * *$ & $* * *$ \\
\hline Age & $0.99(0.97-1.02)$ & NS & NS \\
\hline \multicolumn{4}{|l|}{ Sex } \\
\hline Female & 1.00 & & \\
\hline Male & $0.62(0.50-0.77)$ & $* * *$ & $* * *$ \\
\hline \multicolumn{4}{|l|}{ Twin status } \\
\hline Not a twin & 1.00 & & \\
\hline Monozygotic & $1.20(0.93-1.54)$ & NS & NS \\
\hline Dizygotic & $1.19(0.93-1.52)$ & NS & NS \\
\hline
\end{tabular}

APR adjusted prevalence ratio, NS non-significant $(p>0.05)$.

${ }^{*} p<0.05$

$*^{* *} p<0.01$

*** $p<0.001$

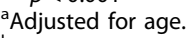

${ }^{\mathrm{b}}$ Adjusted for zygosity.

'Adjusted for sex.
Altogether, we find evidence for general and specific relationships between self-rated mental health symptoms and self-reported impairment and show that somatic symptoms are associated with impairment to a greater degree than anxious-depressive, hypomanic, and psychotic-like symptoms in a community-based young adult sample. Health professionals should be alert to the increased risk of functional impairment in young adults presenting with somatic symptoms.

\section{Acknowledgements}

We thank the participants and their families for engagement with this longitudinal study. The BLTS studies have been supported by NHMRC project grants 1031119 and 1049911 (Cl: Martin). The 19Up follow-up was supported by NHMRC (APP10499110) and the NIH grants (K99R00, RO0DA023549).

\section{Author details \\ ${ }^{1}$ Youth Mental Health \& Technology Team, Brain and Mind Centre, University of Sydney, Sydney, Australia. ${ }^{2}$ Academic Psychiatry, Institute of Neuroscience, Newcastle University, Newcastle, UK. ${ }^{3}$ Diderot University, Paris, France. ${ }^{4}$ Norwegian University of Science and Technology, Trondheim, Norway. ${ }^{5}$ QIMR Berghofer Institute of Medical Research, Brisbane, Australia. ${ }^{6}$ Institute for Molecular Bioscience, The University of Queensland, Brisbane, Australia. ${ }^{7}$ ARAMIS Laboratory, Paris Brain Institute, Paris, France. ${ }^{8}$ Thompson Institute, University of the Sunshine Coast, Birtinya, Australia. ${ }^{9}$ Virginia Institute for Psychiatric and Behavioral Genetics, Virginia Commonwealth University, Virginia, USA}

\section{Data availability}

The data that support the findings of this study were made available to authors via the BLTS research committee (that approved the cohort study). The authors confirm that the summary data for all variables supporting the findings of this study are included within the article and its supplementary materials. The raw data are being used at the lead research centers and form part of an ongoing program of research and data are only made available upon written 
request to the BLTS research committee. Data are not publicly available due to confidentiality restrictions and because research participants did not give permission for dissemination beyond the BLTS research team.

\section{Conflict of interest}

I.B.H. reports personal fees from National Mental Health Commission, personal fees from Medibank Clinical Reference Group, non-financial support from Psychosis Australia Trust, grants from NHMRC, grants from Innowell Pty LTD, grants from NHMRC, grants from NHMRC, outside the submitted work. I.B.H. was an inaugural Commissioner on Australia's National Mental Health Commission (2012-2018). He is the Co-Director, Health and Policy at the Brain and Mind Centre (BMC) University of Sydney. The BMC operates an earlyintervention youth services at Camperdown under contract to headspace. I.B. $\mathrm{H}$. has previously led community-based and pharmaceutical industrysupported (Wyeth, Eli Lily, Servier, Pfizer, AstraZeneca) projects focused on the identification and better management of anxiety and depression. He was a member of the Medical Advisory Panel for Medibank Private until October 2017, a Board Member of Psychosis Australia Trust and a member of Veterans Mental Health Clinical Reference group. He is the Chief Scientific Advisor to, and an equity shareholder in, Innowell. Innowell has been formed by the University of Sydney and PwC to deliver the $\$ 30 \mathrm{~m}$ Australian Governmentfunded "Project Synergy". Project Synergy is a three-year program for the transformation of mental health services through the use of innovative technologies.

\section{Publisher's note}

Springer Nature remains neutral with regard to jurisdictional claims in published maps and institutional affiliations.

Supplementary information The online version contains supplementary material available at https://doi.org/10.1038/s41398-021-01390-y.

Received: 10 November 2020 Revised: 25 March 2021 Accepted: 14 April 2021

Published online: 13 May 2021

\section{References}

1. UN General Assembly. Transforming our world: the 2030 Agenda for Sustainable Development. 21 October 2015, A/RES/70/1, available at: https:/ www.refworld.org/docid/57b6e3e44.html (2015).

2. Gore, F. M. et al. Global burden of disease in young people aged 10-24 years: a systematic analysis. Lancet $\mathbf{3 7 7}, 2093-2102$ (2011).

3. Scott, J. et al. Adolescents and young adults who are not in employment, education, or training. BMJ 347, f5270 (2013).

4. Asselmann, E., Wittchen, H. U., Lieb, R. \& Beesdo-Baum, K. Sociodemographic, clinical, and functional long-term outcomes in adolescents and young adults with mental disorders. Acta Psychiatr. Scand. 137, 6-17 (2018).

5. Angold, A., Costello, E. J., Farmer, E. M., Burns, B. J. \& Erkanli, A. Impaired but undiagnosed. J. Am. Acad. Child Adolesc. Psychiatry 38, 129-137 (1999).

6. Rickwood, D. J., Telford, N. R., Parker, A. G., Tanti, C. J. \& McGorry, P. D. headspace - Australia's innovation in youth mental health: who are the clients and why are they presenting? Med. J. Aust. 200, 1-4 (2014).

7. Scott, E. M. et al. Targeted primary care-based mental health services for young Australians. Med. J. Aust. 196, 136-140 (2012).

8. Broadhead, W. E., Blazer, D. G., George, L. K. \& Tse, C. K. Depression, disability days, and days lost from work in a prospective epidemiologic survey. Jama 264, 2524-2528 (1990).

9. Barsky, A. J., Orav, E. J. \& Bates, D. W. Somatization increases medical utilization and costs independent of psychiatric and medical comorbidity. Arch. Gen. Psychiatry 62, 903-910 (2005)

10. Harris, A. M., Orav, E. J., Bates, D. W. \& Barsky, A. J. Somatization increases disability independent of comorbidity. J. Gen. Intern. Med. 24, 155-161 (2009).

11. Kroenke, K. et al. Physical symptoms in primary care. Predictors of psychiatric disorders and functional impairment. Arch. Fam. Med. 3, 774-779 (1994).

12. Yung, A. R. et al. Psychotic-like experiences in a community sample of adolescents: implications for the continuum model of psychosis and prediction of schizophrenia. Aust. N. Z. J. Psychiatry 43, 118-128 (2009).
13. Armando, M. et al. Psychotic-like experiences and correlation with distress and depressive symptoms in a community sample of adolescents and young adults. Schizophr. Res. 119, 258-265 (2010).

14. Yung, A. R. et al. Psychotic-like experiences in nonpsychotic help-seekers: associations with distress, depression, and disability. Schizophr. Bull. 32, 352-359 (2005)

15. Judd, L. L. \& Akiskal, H. S. The prevalence and disability of bipolar spectrum disorders in the US population: re-analysis of the ECA database taking into account subthreshold cases. J. Affect. Disord. 73, 123-131 (2003).

16. Lewinsohn, P. M., Klein, D. N. \& Seeley, J. R. Bipolar disorder during adolescence and young adulthood in a community sample. Bipolar Disord. 2, 281-293 (2000).

17. Lewinsohn, P. M., Seeley, J. R. \& Klein, D. N. Bipolar disorders during adolescence. Acta Psychiatr. Scand. 108, 47-50 (2003).

18. Balazs, J. et al. Adolescent subthreshold-depression and anxiety: psychopathology, functional impairment and increased suicide risk. J. Child Psychol. Psychiatry Allied Discip. 54, 670-677 (2013).

19. Cuijpers, P., de Graaf, R. \& van Dorsselaer, S. Minor depression: risk profiles, functional disability, health care use and risk of developing major depression. J. Affect. Disord. 79, 71-79 (2004)

20. Judd, L. L., Schettler, P. J. \& Akiskal, H. S. The prevalence, clinical relevance, and public health significance of subthreshold depressions. Psychiatr. Clin. N. Am. 25, 685-698 (2002).

21. Kessler, R. C., Zhao, S., Blazer, D. G. \& Swartz, M. Prevalence, correlates, and course of minor depression and major depression in the National Comorbidity Survey. J. Affect. Disord. 45, 19-30 (1997).

22. Rai, D., Skapinakis, P., Wiles, N., Lewis, G. \& Araya, R. Common mental disorders, subthreshold symptoms and disability: Iongitudinal study. Br. J. Psychiatry 197 411-412 (2010).

23. Addington, J., Penn, D., Woods, S. W., Addington, D. \& Perkins, D. O. Social functioning in individuals at clinical high risk for psychosis. Schizophr. Res. 99, 119-124 (2008).

24. Scott, J. et al. Prevalence of self-reported subthreshold phenotypes of major mental disorders and their association with functional impairment, treatment and full-threshold syndromes in a community-residing cohort of young adults. Early Interv. Psychiatry 15, 306-313 (2020).

25. Shah, J. L. et al. Transdiagnostic clinical staging in youth mental health: a first international consensus statement. World Psychiatry 19, 233-242 (2020).

26. Krueger, R. F. \& Markon, K. E. Reinterpreting comorbidity: a model-based approach to understanding and classifying psychopathology. Annu. Rev. Clin. Psychol. 2, 111-133 (2006).

27. Wittchen, H.-U. Epidemiological research in mental disorders: lessons for the next decade of research-the NAPE Lecture 1999. Acta Psychiatr. Scand. 101, 2-10 (2000).

28. Preisig, M., Merikangas, K. R. \& Angst, J. Clinical significance and comorbidity of subthreshold depression and anxiety in the community. Acta Psychiatr. Scand. 104, 96-103 (2001).

29. Alonso, J. et al. Days out of role due to common physical and mental conditions: results from the WHO World Mental Health surveys. Mol. Psychiatry 16, 1234-1246 (2011)

30. Ormel, J. et al. Common mental disorders and disability across cultures. Results from the WHO Collaborative Study on Psychological Problems in General Health Care. Jama 272, 1741-1748 (1994).

31. Slade, T., Johnston, A., Oakley Browne, M. A., Andrews, G. \& Whiteford, H. 2007 National Survey of Mental Health and Wellbeing: methods and key findings. Aust. N. Z. J. Psychiatry 43, 594-605 (2009).

32. Hickie, I. B. et al. Development of a simple screening tool for common mental disorders in general practice. Med. J. Aust. 175, S10-S17 (2001).

33. Ormel, J. et al. Disability and treatment of specific mental and physical disorders across the world. Br. J. Psychiatry 192, 368-375 (2008).

34. Elm, E. V. et al. Strengthening the reporting of observational studies in epidemiology (STROBE) statement: guidelines for reporting observational studies. BMJ 335, 806-808 (2007).

35. Gillespie, N. A. et al. The Brisbane longitudinal twin study: pathways to Cannabis use, abuse, and dependence project-current status, preliminary results, and future directions. Twin Res. Hum. Genet. 16, 21-33 (2013).

36. Wright, M. J. \& Martin, N. G. Brisbane Adolescent Twin Study: outline of study methods and research projects. Aust. J. Psychol. 56, 65-78 (2004).

37. Coury-Duchesne, B. et al. Nineteen and Up study (19Up): understanding pathways to mental health disorders in young Australian twins. BMJ Open $\mathbf{8}$ e018959 (2018). 
38. Wright, M. et al. Genetics of cognition: outline of a Collaborative Twin Study. Twin Res. 4, 48-56 (2001).

39. Scott, J. et al. Can network analysis of self-reported psychopathology shed light on the core phenomenology of bipolar disorders in adolescents and young adults? Bipolar Disorders https://doi.org/10.1111/bdi.13067 (2021) Epub ahead of print.

40. Mitchell, B. L. et al. Twenty-five and up (25Up) study: a new wave of the Brisbane Longitudinal Twin Study. Twin Res. Hum. Genet. 22, 154-163 (2019).

41. Hadzi-Pavlovic, D. et al. Screening for prolonged fatigue syndromes: validation of the SOFA scale. Soc. Psychiatry Psychiatr. Epidemiol. 35, 471-479 (2000).

42. Goldberg, D. P. Manual of the General Health Questionnaire. (NFER Publishing, 1978).

43. Bechdolf, A. et al. The predictive validity of bipolar at-risk (prodromal) criteria in help-seeking adolescents and young adults: a prospective study. Bipolar Disord. 16, 493-504 (2014).

44. Scott, J. et al. Bipolar at-risk criteria: an examination of which clinical features have optimal utility for identifying youth at risk of early transition from depression to bipolar disorders. Schizophr. Bull. 43, 737-744 (2017).

45. Altman, E. G., Hedeker, D., Peterson, J. L. \& Davis, J. M. The Altman self-rating mania scale. Biol. Psychiatry 42, 948-955 (1997).

46. Angst, J. et al. Toward a re-definition of subthreshold bipolarity: epidemiology and proposed criteria for bipolar-ll, minor bipolar disorders and hypomania. J. Affect. Disord. 73, 133-146 (2003).

47. Kessler, R. C. et al. Validity of the assessment of bipolar spectrum disorders in the WHO CIDI 3.0. J. Affect. Disord. 96, 259-269 (2006).

48. Merikangas, K. R. et al. Lifetime and 12-month prevalence of bipolar spectrum disorder in the National Comorbidity Survey Replication. Arch. Gen. Psychiatry 64, 543-552 (2007).

49. Ustun, T. B. et al. Developing the World Health Organization Disability Assessment Schedule 2.0. Bull. World Health Organ. 88, 815-823 (2010).

50. Revicki, D. A., Irwin, D., Reblando, J. \& Simon, G. E. The accuracy of self-reported disability days. Med. Care 32, 401-404 (1994).

51. R Core Team. R: A Language and Environment for Statistical Computing ( $R$ Foundation for Statistical Computing, Vienna, Austria, 2018) Available online at https://www.R-project.org/.

52. Santos, C. A. S. T. et al. Estimating adjusted prevalence ratio in clustered crosssectional epidemiological data. BMC Med. Res. Methodol. 8, 80 (2008).

53. de Oliveira, N. F., Santana, V. S. \& Lopes, A. A. Ratio of proportions and the use of the delta method for confidence intervals in logistic regression. Rev. de. Saude Publica 31, 90-99 (1997).

54. Benjamini, Y. \& Hochberg, Y. Controlling the false discovery rate: a practical and powerful approach to multiple testing. J. R. Stat. Soc. Ser. B 57, 289-300 (1995).

55. Rask, C. U. et al. Functional somatic symptoms and associated impairment in 5-7-year-old children: the Copenhagen Child Cohort 2000. Eur. J. Epidemiol. 24, 625-634 (2009).

56. Lieb, R., Pfister, H., Mastaler, M. \& Wittchen, H.-U. Somatoform syndromes and disorders in a representative population sample of adolescents and young adults: prevalence, comorbidity and impairments. Acta Psychiatr. Scand. 101, 194-208 (2000).
57. van Geelen, S. M., Rydelius, P.-A. \& Hagquist, C. Somatic symptoms and psychological concerns in a general adolescent population: exploring the relevance of DSM-5 somatic symptom disorder. J. Psychosom. Res. 79, 251-258 (2015).

58. Hoedeman, R., Blankenstein, A. H., Krol, B., Koopmans, P. C. \& Groothoff, J. W. The contribution of high levels of somatic symptom severity to sickness absence duration, disability and discharge. J. Occup. Rehabil. 20, 264-273 (2010).

59. Judd, L. L. et al. Psychosocial disability in the course of bipolar I and II disorders: a prospective, comparative, longitudinal study. Arch. Gen. Psychiatry $6 \mathbf{6 2}$ 1322-1330 (2005).

60. Bowie, C. R. et al. Prediction of real-world functional disability in chronic mental disorders: A comparison of schizophrenia and bipolar disorder. Am. J. Psychiatry 167, 1116-1124 (2010).

61. Crouse, J. J. et al. Modelling associations between neurocognition and functional course in young people with emerging mental disorders: a longitudinal cohort study. Transl. Psychiatry 10, 1-9 (2020).

62. Kelleher, I. et al. Psychotic experiences in the population: association with functioning and mental distress. Schizophr. Res. 165, 9-14 (2015).

63. Oh, H., Koyanagi, A., Kelleher, I. \& DeVylder, J. Psychotic experiences and disability: findings from the Collaborative Psychiatric Epidemiology Surveys. Schizophr. Res. 193, 343-347 (2018).

64. Henningsen, P., Zipfel, S. \& Herzog, W. Management of functional somatic syndromes. Lancet 369, 946-955 (2007).

65. Kroenke, K. A practical and evidence-based approach to common symptoms: a narrative review. Ann. Intern. Med. 161, 579-586 (2014).

66. Kroenke, K. Patients presenting with somatic complaints: epidemiology, psychiatric comorbidity and management. Int. J. Methods Psychiatr. Res. 12, 34-43 (2003).

67. Kredlow, M. A., Capozzoli, M. C., Hearon, B. A., Calkins, A. W. \& Otto, M. W. The effects of physical activity on sleep: a meta-analytic review. J. Behav. Med. 38, 427-449 (2015).

68. Kaplan, K. A. \& Harvey, A. G. Hypersomnia across mood disorders: a review and synthesis. Sleep Med. Rev. 13, 275-285 (2009).

69. Ablin, J. N. et al. Effects of sleep restriction and exercise deprivation on somatic symptoms and mood in healthy adults. Clin. Exp. Rheumatol. 31, S53-S59 (2013).

70. Freeman, D. et al. The effects of improving sleep on mental health (OASIS): a randomised controlled trial with mediation analysis. Lancet Psychiatry 4, 749-758 (2017).

71. Freeman, D., Sheaves, B., Waite, F., Harvey, A. G. \& Harrison, P. J. Sleep disturbance and psychiatric disorders. Lancet Psychiatry 7, 628-637 (2020).

72. Bellivier, F., Geoffroy, P. A., Etain, B. \& Scott, J. Sleep- and circadian rhythmassociated pathways as therapeutic targets in bipolar disorder. Expert Opin. Ther. Targets 19, 747-763 (2015).

73. Crouse, J. J. et al. Circadian rhythm sleep-wake disturbances and depression in young people: implications for prevention and early intervention. Lancet Psychiatry (In Press).

74. Carpenter, J. S. et al. Circadian depression: a mood disorder phenotype. Neurosci. Biobehav. Rev. 126, 79-101 (2021). 